\title{
Viabilidade de formulação peletizada do fungo nematófago Monacrosporium sinense, no controle biológico de nematóides parasitos gastrintestinais de bezerros
}

\author{
[Viability of pellet formulation of Monacrosporium sinense as biological control of \\ gastrointestinal nematodes of calves]

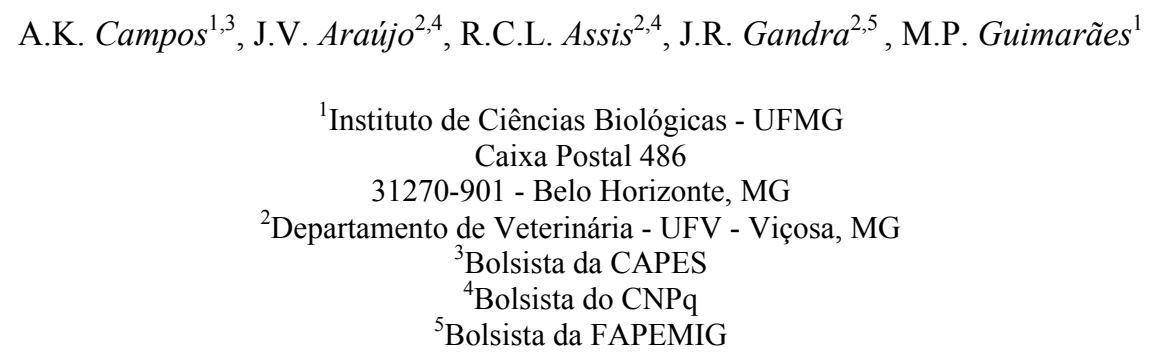

\section{RESUMO}

A viabilidade de uma formulação do fungo Monacrosporium sinense foi avaliada no controle de nematóides parasitos gastrintestinais de bovinos. Dois grupos de 10 bezerros cada um, mestiços Holandês x Zebu, de seis a nove meses de idade, foram colocados em pastagem de Brachiaria brizantha. Em um dos grupos, cada animal recebeu $20 \mathrm{~g}$ de péletes em matriz de alginato de sódio, contendo massa miceliana do fungo $M$. sinense via oral, duas vezes por semana, durante seis meses, com início no mês de outubro; no outro grupo, controle, os bezerros não receberam esse tratamento. As contagens de ovos por grama de fezes $(\mathrm{OPG})$ e de larvas infectantes por $\mathrm{kg}$ de matéria seca foram maiores $(\mathrm{P}<0,05)$ no grupocontrole, e a diferença entre o OPG dos dois grupos, no final do experimento, foi de $79 \%$. A viabilidade dos péletes em germinar e a atividade predatória do fungo após o encapsulamento foram avaliadas in vitro. A porcentagem de péletes com cultivo positivo para o fungo variou entre 90 e $100 \%$, e o percentual de redução de larvas infectantes in vitro variou entre 90,6 e 100\%. A aplicação de péletes de M. sinense na dosagem e periodicidade usadas são eficazes no biocontrole de nematóides parasitos gastrintestinais de bovinos.

Palavras-chave: nematóides, controle biológico, fungos nematófagos, Monacrosporium sinense

\begin{abstract}
The viability of a formulation of the fungus Monacrosporium sinense was evaluated as control of bovine gastrointestinal nematodes parasites. Two groups were used and they were made up of 10 Holstein X Zebu crossbred, six to eight-month-old. They were grazing on Brachiaria brizantha pasture. In the treated group, each animal received orally, twice a week $20 \mathrm{~g}$ of pellets of sodium alginate containing mycelial of the fungus M. sinense, during six months, with the onset in October. In the control group, the calves did not receive that treatment. The counting of eggs per gram of faeces (EPG) and the counting of infective larvae per $\mathrm{kg}$ of dry matter were higher $(P<0.05)$ in the control group than in the treated group. The difference of the EPG between the groups at the end of the experimental period was 79\%. The viability of the pellets germination and the predatory activity of the fungus after the encapsulation were evaluated in vitro. The percentage of pellets with positive culture for fungus varied between $90-100 \%$ and the percentage of reduction of infective larvae varied between $90.6-100 \%$. The use of that dose and the periodic application of $\mathrm{M}$. sinense pellets were efficient as control of bovine gastrointestinal nematode parasites.
\end{abstract}

Keywords: nematodes, biological control, nematophagous fungi, Monacrosporium sinense

Recebido em 14 de fevereiro de 2005

Aceito em 21 de novembro de 2006

E-mail: arturkanadani@hotmail.com

Apoio: FAPEMIG/CNPq 


\section{INTRODUÇÃO}

O parasitismo gastrintestinal por nematóides tricostrongilídeos constitui um relevante problema nos rebanhos bovinos mundiais. Seu controle fundamenta-se, principalmente, na administração de drogas anti-helmínticas de amplo espectro de ação. Problemas como resistência às drogas (Fiel et al., 2001; Paiva et al. 2001), resíduos químicos em produtos alimentícios de origem animal e ecotoxicidade (Herd, 1993) têm incentivado a busca de métodos alternativos para o controle desses parasitos em bovinos. O controle biológico com uso de fungos nematófagos é o método que tem apresentado resultados mais promissores (Waller e Larsen, 1993). Esses fungos predam nematóides através de estruturas especializadas, denominadas armadilhas.

No Brasil, estudos preliminares desenvolvidos por Araújo (1996) e Gomes (1998) relataram o uso de fungos nematófagos no controle da infestação das pastagens por larvas de nematóides tricostrongilídeos de bovinos. Entre as diversas espécies, as pertencentes ao gênero Monacrosporium têm sido alvo de diversos estudos (Gomes et al., 1999; Araújo et al., 2000; Araújo et al., 2004ab). Os fungos do gênero Monacrosporium foram classificados por Cooke e Dickson como pertencentes à subdivisão Deuteromycotina. A espécie Monacrosporium sinense preda larvas de nematóides através de redes adesivas, produz conídios que medem entre $25-30 \mu \mathrm{m}$ de comprimento por $15-18 \mu \mathrm{m}$ de largura e clamidósporos com $20-24 \mu \mathrm{m}$ de comprimento por $17-27 \mu \mathrm{m}$ de largura.

Alguns estudos foram realizados com o objetivo de avaliar isolados fúngicos dessa espécie no biocontrole de nematóides parasitos gastrintestinais de animais domésticos (Araújo e Ribeiro, 2003; Araújo et al., 2004ab).

Até o momento, não existe nenhuma formulação fúngica para o controle biológico de nematódeos gastrintestinais de bovinos disponível no mercado. Experimentalmente, algumas técnicas de encapsulamento têm sido testadas no desenvolvimento de uma formulação que viabilize o emprego desses agentes de biocontrole (Walker e Connick, 1983; Araújo et al., 1996; Araújo et al., 1999; Waller et al., 2001; Campos, 2002).
O objetivo deste trabalho foi avaliar a viabilidade de uma formulação peletizada em matriz de alginato de sódio do fungo predador de nematóides Monacrosporium sinense e verificar sua eficácia no controle de nematóides gastrintestinais em bovinos.

\section{MATERIAL E MÉTODOS}

Uma amostra de Monacrosporium sinense (SF 53), isolado de solos brasileiros, oriundo da micoteca do Departamento de Veterinária da Universidade Federal de Viçosa, mantido em tubos de ensaio em geladeira a $4^{\circ} \mathrm{C}$, foi repicado e crescido em meio GPL (15g de glicose, $2 \mathrm{~g}$ de peptona, $5 \mathrm{~g}$ de extrato de levedura, $0,5 \mathrm{~g}$ de $\mathrm{K}_{2} \mathrm{HPO}_{4}, 0,25 \mathrm{~g}$ de $\mathrm{MgSO}_{4} .7 \mathrm{H}_{2} \mathrm{O}$ ), contido em frascos Erlenmayers, sob agitação de 120rpm, em câmara incubadora regulada a $25^{\circ} \mathrm{C}$.

Péletes contendo massa miceliana do fungo $M$. sinense, constituídos em matriz de alginato de sódio, foram produzidos ao início de cada mês do período experimental, de acordo com a técnica descrita por Walker e Connick (1983) e modificada por Lackey et al. (1993). Os péletes foram embalados em sacos plásticos transparentes e conservados em geladeira a $4^{\circ} \mathrm{C}$.

Após a confecção dos péletes, a cada mês testou$\mathrm{se}$, in vitro, a habilidade dos fungos em crescer e predar nematóides. Cinqüenta péletes, 10 por placa, foram distribuídos sobre placas de Petri ( $9 \mathrm{~cm}$ de diâmetro) contendo $20 \mathrm{ml}$ de ágar-água. As placas foram incubadas em estufa BOD a $25^{\circ} \mathrm{C}$, e o crescimento de colônias, a partir de cada pélete, foi observado durante cinco dias, após o que se calculou o percentual de péletes a partir dos quais houve formação de colônias. Aproximadamente 1000 larvas de Haemonchus contortus foram gotejadas sobre as placas. Essas foram mantidas em estufa a $25^{\circ} \mathrm{C}$ e observadas em microscópio (100x), diariamente, por cinco dias. Foram utilizadas, como controle, placas de ágar-água sem fungo sobre as quais foram gotejadas, aproximadamente, 1000 larvas infectantes de $H$. contortus. As larvas, livres de predação foram recuperadas mediante lavagem da superfície da placa, com $5 \mathrm{ml}$ de água e auxílio de espátula metálica. O percentual de redução de larvas do grupo tratado em relação ao controle foi calculado pela fórmula: 


$$
\% \text { redução }=\frac{X_{c}-X_{t}}{X_{c}} \cdot x 100, \text { em que: }
$$

$\mathrm{X}_{\mathrm{c}}=$ número de larvas recuperadas das placas controle e $\mathrm{X}_{\mathrm{t}}=$ número de larvas recuperadas das placas tratadas.

O teste de controle biológico in vivo foi realizado em uma fazenda localizada no município de Viçosa, estado de Minas Gerais no período de outubro de 2003 a março de 2004. O grupo de animais foi constituído por 20 bezerros, 16 fêmeas e quatro machos, mestiços Holandês $\mathrm{x}$ Zebu, com seis a nove meses de idade. Esses animais foram tratados com o anti-helmíntico albendazol na dose de 7,5mg por quilograma de peso vivo.

Após sete dias do tratamento, os bezerros foram divididos em dois grupos de 10 animais cada um, oito fêmeas e dois machos, em dois piquetes de Brachiaria brizantha, com, aproximadamente, quatro hectares, naturalmente infestados com larvas infectantes $\left(\mathrm{L}_{3}\right)$, devido ao prévio pastejo por bezerros infectados, formando um grupo tratado com a formulação contendo o fungo $M$. sinense e outro controle. No primeiro grupo, cada animal recebeu $20 \mathrm{~g}$ de péletes contendo aproximadamente 2-2,5g de micélio do fungo $M$. sinense, duas vezes por semana, durante seis meses, a partir do começo do período chuvoso na região. No grupo-controle, os animais não receberam péletes com fungo.

Semanalmente, dos animais de cada grupo, foram colhidas amostras de fezes diretamente do reto, para determinação da contagem de ovos por grama de fezes (OPG), segundo a técnica modificada de Gordon e Whitlock (1939), descrita por Lima (1989). Paralelamente ao exame de OPG, foram realizadas coproculturas, em que $20 \mathrm{~g}$ de fezes, misturadas com carvão vegetal fragmentado e umedecidas, foram levadas à estufa a $26^{\circ} \mathrm{C}$, durante oito dias, para obtenção de larvas de nematóides parasitos gastrintestinais. Essas larvas foram identificadas de acordo com os critérios estabelecidos por Keith (1953).

Amostras de $3 \mathrm{~g}$ de fezes foram coletadas dos animais para $\mathrm{o}$ isolamento de fungos nematófagos. As fezes foram semeadas em placas contendo ágar-água $2 \%$, acrescidas de 2000 larvas de Panagrellus sp e incubadas em estufa a $25^{\circ} \mathrm{C}$, durante 21 dias. Após esse período, as placas foram observadas com o auxílio de um microscópio estereoscópico para visualização de armadilhas e esporos do fungo. Os fungos isolados foram identificados utilizando-se as chaves de Cooke e Godfrey (1964), Van Ooorschot (1985) e Liu e Zhang (1994).

Uma vez por semana, em cada piquete, foram coletadas amostras de pastagem, em ziguezague, de pontos variados. De cada amostra, foram utilizados em torno de $500 \mathrm{~g}$ de pastagem, de onde se recuperaram as larvas de nematóides parasitos de bovinos, segundo técnica descrita por Lima (1989). As amostras de pastagem foram colocadas em uma estufa de secagem a $100^{\circ} \mathrm{C}$ até que atingissem peso constante, para se obter o peso da matéria seca. Os dados obtidos foram transformados em número de larvas por $\mathrm{kg}$ de matéria seca.

Foram registradas, diariamente, as médias de temperatura, umidade relativa do ar e precipitação pluvial, colhidos em uma estação especializada na região de Viçosa-MG.

Os resultados de OPG e número de larvas recuperadas da pastagem foram transformados em Log de $\mathrm{X}+1$, e submetidos à análise de variância. As médias foram comparadas pelo teste Tukey em nível de $5 \%$ de probabilidade.

\section{RESULTADOS}

A formulação contendo o fungo $M$. sinense e alginato de sódio demonstrou alta viabilidade in vitro durante todo o período experimental. A porcentagem de péletes com cultivo positivo para o fungo variou entre 90 e $100 \%$ (Tab. 1), e o percentual de redução de larvas infectantes in vitro variou entre 90,6 e $100 \%$. Nas placas inoculadas com os péletes, foram observadas intensa esporulação do fungo e presença de larvas capturadas em armadilhas do fungo.

O OPG dos animais do grupo-controle foi maior $(\mathrm{P}<0,05)$ do que o dos animais do grupo tratado nos meses de janeiro, fevereiro e março de 2004 (Fig. 1). Durante os meses de outubro, novembro e dezembro, o gênero Cooperia foi o mais prevalente nas coproculturas dos dois grupos experimentais. Nos meses subseqüentes, a 
ocorrência de Haemonchus e Oesophagostomum superou a de Cooperia, e a de Haemonchus, sempre foi maior que a de Oesophagostomum. A média das porcentagens de larvas ao final do experimento indica maior prevalência do gênero Haemonchus (43,8\%), seguido dos gêneros Cooperia (35,1\%) e Oesophagostomum (14,9\%).

O número de larvas totais recuperadas no piquete dos animais do grupo tratado com a formulação foi menor 50,1\% $(\mathrm{P}<0,05)$ em relação ao grupo- controle (Fig. 2). Quando se avaliou a redução da contaminação da pastagem do grupo tratado no segundo trimestre de experimento, isoladamente, o percentual de redução foi de $58,6 \%$. Os gêneros Cooperia e Haemonchus foram recuperados das pastagens dos dois grupos durante todo o período experimental, sendo o gênero Cooperia o mais prevalente. $\mathrm{O}$ gênero Oesophagostomum foi recuperado somente a partir do mês de dezembro.

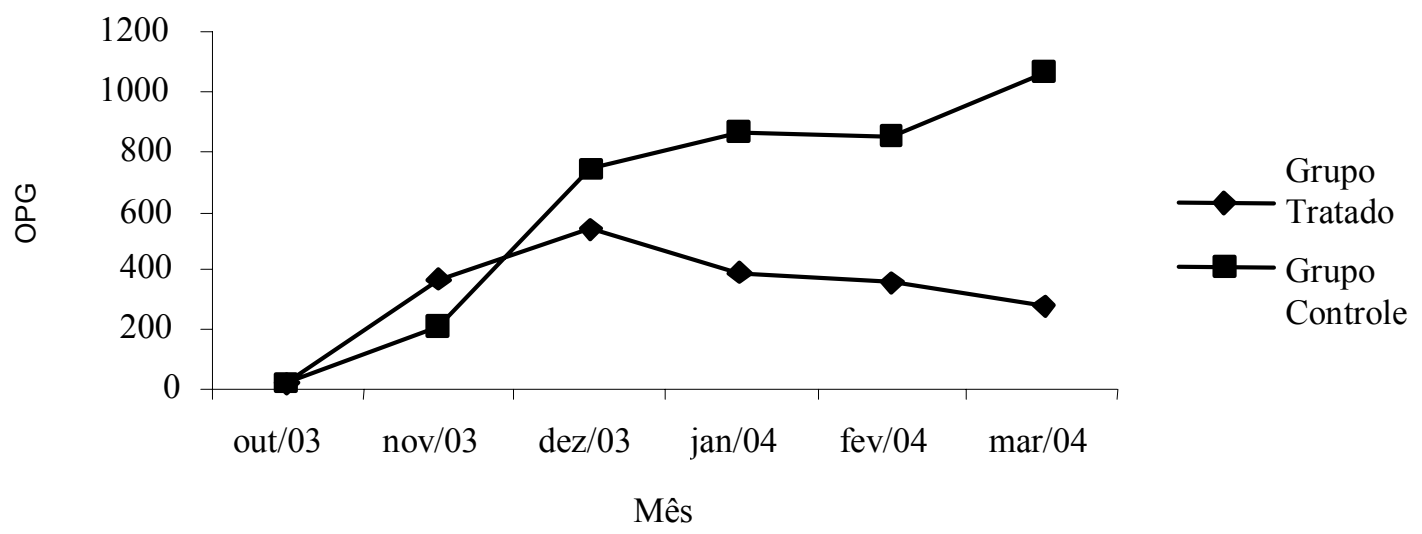

Figura 1. Valores médios do número de ovos de nematóides por grama de fezes (OPG) recuperados das coproculturas de bezerros do grupo tratado com péletes do fungo Monacrosporium sinense (grupo A) e do grupo-controle (grupo B), na região de Viçosa-MG, durante o período de outubro de 2003 a março de 2004.

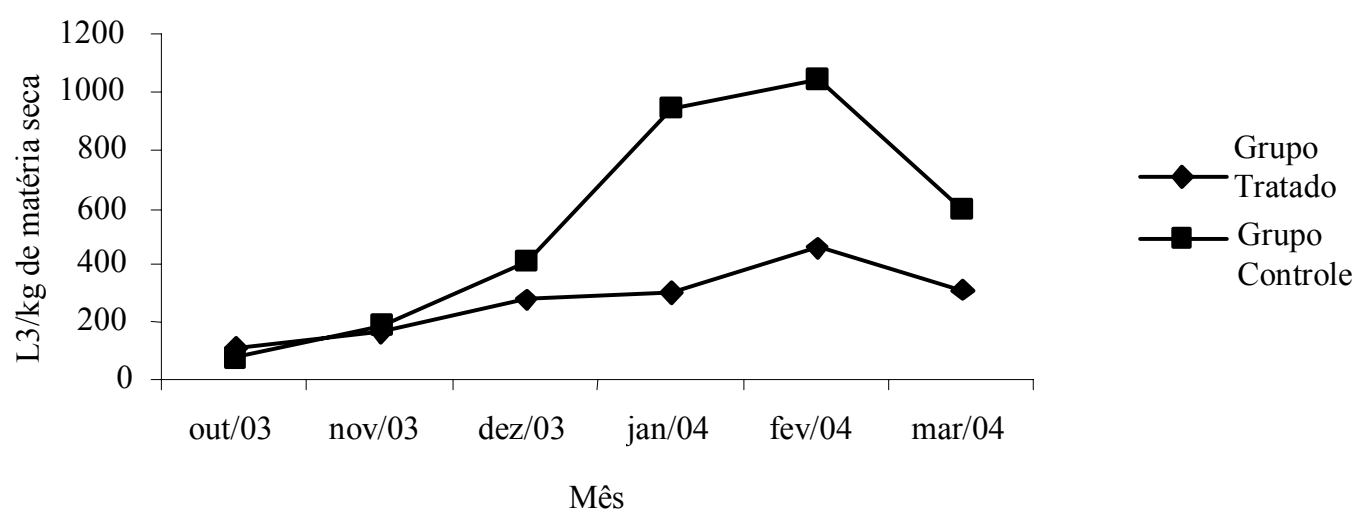

Figura 2. Médias das contagens de larvas infectantes de nematóides por kg de matéria seca de pastagem coletadas em piquetes com bezerras do grupo tratado com péletes de alginato contendo o fungo predador Monacrosporium sinense (grupo A) e do grupo-controle (grupo B) na região de Viçosa-MG, durante o período de outubro de 2003 a março de 2004. 
Durante o período experimental, as temperaturas médias mensais oscilaram entre $20,7^{\circ} \mathrm{C}$ (outubro de 2003) e $22,9^{\circ} \mathrm{C}$ (dezembro de 2003). A precipitação pluvial mensal oscilou entre 27,8mm (outubro de 2003) e 405,9 mm (mês de janeiro de 2004).

O fungo $M$. sinense foi isolado em placas inoculadas com fezes dos bezerros do grupo tratado, nos meses de outubro e março. Neste último mês, foi também isolado um fungo identificado como Arthrobotrys musiformis (Tab. 1). Nas placas do grupo-controle, no mês de outubro de 2003, foi encontrado um isolado do gênero Arthrobotrys sp. e, no mês de fevereiro de 2004, um isolado não identificado devido à ausência de estruturas que permitissem sua identificação.

Tabela 1. Viabilidade de péletes de alginato de sódio contendo massa micelial do fungo Monacrosporium sinense após 5 dias de incubação a $25^{\circ} \mathrm{C}$ e percentual de redução do número de larvas de Haemonchus contortus

\begin{tabular}{lcc}
\hline Mês & Viabilidade & $\begin{array}{c}\text { Redução por } \\
\text { ensaio }\end{array}$ \\
\hline Outubro & $100 \%$ & $98,3 \%$ \\
Novembro & $100 \%$ & $100 \%$ \\
Dezembro & $100 \%$ & $90,6 \%$ \\
Janeiro & $90 \%$ & $99,3 \%$ \\
Fevereiro & $100 \%$ & $100 \%$ \\
Março & $100 \%$ & $93,6 \%$ \\
\hline
\end{tabular}

Ao final do período experimental, não foram detectadas diferenças $(\mathrm{P}>0,05)$ no peso dos animais do grupo tratado em relação aos do grupo controle $\mathrm{O}$ ganho de peso do grupo tratado durante o período experimental foi de $71,1 \mathrm{~kg}$ e do grupo-controle foi de $75,7 \mathrm{~kg}$.

\section{DISCUSSÃO}

Fungos do gênero Monacrosporium têm sido alvo de diversos estudos, com o objetivo de avaliar sua aplicação em programas de controle biológico de nematóides tricostrongilídeos de ruminantes.

Araújo et al. (2000) observaram viabilidade de péletes de um isolado de Monacrosporium thaumasium durante 16 semanas à temperatura de $4^{\circ} \mathrm{C}$ em sal mineral.

A alta viabilidade in vitro dos péletes durante o experimento demonstrou que o isolado de $M$. sinense aqui utilizado sobrevive aos processos de encapsulamento em alginato de sódio, entretanto estudos complementares são necessários para avaliar a viabilidade dessa formulação por longos períodos de conservação e tornar sua aplicação uma realidade no biocontrole de nematóides parasitos de animais.
A redução da contaminação da pastagem com $58,6 \%$ nos três últimos meses de experimento refletiu no OPG dos animais tratados, que, no mês de março de 2004, foi reduzido em 79\%. A utilização de fungos nematófagos no controle biológico de nematóides parasitos pode reduzir a contaminação da pastagem, agindo diretamente sobre as larvas infectantes no ambiente. Alves et al. (2003) administraram péletes de alginato de sódio contendo $M$. thaumasium para o controle de nematóides gastrintestinais de bovinos criados a campo. Os autores observaram, após seis meses de experimento, redução significativa no número de $\mathrm{L}_{3}$ no piquete dos animais tratados, resultando na redução de $88,8 \%$ desses animais.

Araújo et al. (2004b), ao testarem a mesma formulação, observaram que o tratamento dos animais com os péletes de $M$. thaumasium diminuiu a contaminação da pastagem, reduzindo o OPG dos animais em até $100 \%$, o que tornou os tratamentos anti-helmínticos desnecessários.

As condições climáticas para o desenvolvimento das larvas foram favoráveis durante a maior parte do período experimental (Williams e Mayhew, 1967). Somente no mês de outubro não foi atingida a precipitação pluviométrica mínima $(50 \mathrm{~mm})$ para o desenvolvimento ótimo das formas infectantes dos nematóides tricostrongilídeos. 
A predominância das larvas infectantes de Cooperia e Haemonchus em relação a Oesophagostomum, recuperadas da pastagem, durante o experimento, pode estar relacionada à melhor adaptação às condições ambientais apresentadas. Lima (1989) sugere que os gêneros Cooperia e Haemonchus são os que melhor se adaptam ao desenvolvimento e à sobrevivência nas pastagens, seguidos de Oesophagostomum e outros gêneros.

As temperaturas ideais para crescimento de fungos nematófagos encontram-se entre 20 e $30^{\circ} \mathrm{C}$ (Pandey, 1973) e, segundo Saumell e Padilha (2000), altas precipitações pluviais favoreceriam o seu crescimento no ambiente. Dessa forma, as condições climáticas observadas durante o experimento foram também favoráveis ao desenvolvimento e à eficácia do fungo testado.

O isolamento de $M$. sinense de fezes frescas de bovinos somente nos meses de outubro de 2003 e março de 2004 pode ser justificado pela baixa sensibilidade da técnica de isolamento, como relatado por Manuelli et al. (1999), e pelos tempos de coleta de amostras de fezes que podem também não estar relacionados aos horários de eliminação do fungo após administração da formulação.

A não diferença no ganho de peso de bezerros já foi observada em outros experimentos com fungos utilizados em biocontrole de nematóides (Rabelo, 2000) e pode ser atribuída à baixa carga parasitária, observada nos animais dos grupos tratado e controle, e também à curta duração do ensaio experimental.

Um dos maiores desafios para a implementação do controle biológico de nematóides é o desenvolvimento de formulações com baixo custo, fácil aplicação, alta eficácia e adaptação aos processos de produção industrial (Araújo et al., 2000).

Os resultados deste estudo mostram que o uso de formulação em alginato de sódio contendo o fungo $M$. sinense pode se tornar uma importante alternativa no controle de nematóides tricostrongilídeos parasitos de bovinos.

A utilização dos fungos nematófagos no controle biológico de nematóides é uma alternativa auxiliar ao controle químico e deve ser aplicada não somente quando existe a possibilidade de maior infestação da pastagem por ovos e larvas, mas também quando existir melhores condições para o desenvolvimento do fungo no ambiente, prevenindo, assim, o parasitismo clínico e as perdas de produtividade (Waller e Larsen, 1993). Dessa forma, estudos com este isolado de $M$. sinense em outras épocas do ano, outras regiões com condições climáticas diferentes, deverão ser realizados para permitir sua implementação em programas de controle biológico.

Outra possibilidade é a associação do fungo com outros isolados já testados como forma de aumentar a eficácia de controle.

\section{REFERÊNCIAS BIBLIOGRÁFICAS}

ALVES, P.H.; ARAÚJO, J.V.; GUIMARÃES, M. P. et al. Aplicação de formulação do fungo predador de nematóides Monacrosporium thaumasium no controle de nematóides de bovinos. Arq. Bras. Med. Vet. Zootec., v.55, p.568-573, 2003.

ARAÚJO, J.V. Interação entre larvas infectantes de Cooperia punctata e fungos predadores do gênero Arthrobotrys, caracterização de isolados de Arthrobotrys e seu uso no controle biológico de nematódeos parasitos gastrintestinais de bovinos. 1996. 110f. Tese (Doutorado) - Instituto de Ciências Biológicas, Universidade Federal de Minas Gerais, Belo Horizonte.

ARAÚJO, J.V.; ASSIS, R.C. L.; ALVES, P.H. et al. Controle biológico de tricostrongilídeos (Nematoda:Trichostrongyloidea) gastrintestinais de bovinos pelo fungo Monacrosporium sinense. Arq. Bras. Med. Vet. Zootec., v.56, p.467-471, 2004a.

ARAÚJO, J.V.; GUIMARÃES, M. P.; CAMPOS, A.K. et al. Control of bovine gastrointestinal nematodes parasites using pellets of the nematodetrapping fungus Monacrosporium thaumasium. Ciênc. Rural, v.34, p.457-463, $2004 \mathrm{~b}$.

ARAÚJO, J.V.; NETO, A.P.; AZEVEDO, M.H.F. Screening parasitic nematode- trapping fungi Arthrobotrys for passage through the gastrointestinal tract of calves. Arq. Bras. Med. Vet. Zootec.,v.48, p.543-552, 1996.

ARAUJO, J. V.; RIBEIRO, R. R. Atividade predatória sobre larvas de tricostrongilídeos (Nematoda: Trichostrongyloidea) de isolados fúngicos do gênero Monacrosporium após a passagem pelo trato gastrintestinal de bovinos. Rev. Bras. Parasitol. Vet., v. 12, p. $76-81,2003$. 
ARAÚJO, J.V.; SAMPAIO, W.M.; VASCONCELLOS, R.S. et al. Effects of different temperatures and mineral salt on "pellets" of Monacrosporium thaumasium - a nematode-trapping fungus. Vet. Arhiv., v.70, p.181-190, 2000.

ARAÚJO, J.V.; STEPHANO, M.A.; SAMPAIO, W.M. Passage of nematode trapping fungi through the gastrointestinal tract of calves. Vet. Arhiv., v.69, p. 6978,1999 .

CAMPOS, A.K. Efeito da criopreservação e de formulações sobre a viabilidade do fungo nematófago Monacrosporium spp. 2002. 48f. Dissertação (Mestrado) - Departamento de Veterinária, Universidade Federal de Viçosa, Viçosa, MG.

COOKE, R.C.; GODFREY, B.E.S. A key to the nematode destroying fungi. Trans. Br. Mycol. Soc., v.47, p.61-74, 1964.

FIEL, C.A.; SAUMELL, C.A.; STEFFAN P.E. et al. Resistance of Cooperia to ivermectin treatments in grazing cattle of the Humid Pampa, Argentina. Vet. Parasitol., v.97, p.213-219, 2001.

GOMES, A.P.S. Controle biológico in vivo de nematódeos parasitos gastrintestinais de bovinos pelo fungo Arthrobotrys robusta e atividade in vitro de isolados do fungo Monacrosporium sobre nematódeos. 1998. 81f. Dissertação (Mestrado) Departamento de Veterinária, Universidade Federal de Viçosa, Viçosa, MG.

GOMES, A.P.S.; ARAÚJO, J.V.; RIBEIRO, R.C.F. Differential in vitro pathogenicity of predatory fungi of the genus Monacrosporium for phytonematodes, free-living nematodes and parasitic nematodes of cattle. Braz. J. Med. Biol. Res., v.32, p.79-83, 1999.

GORDON, H. M.; WHITLOCK, H. V. A new technique for counting nematode eggs in sheep faeces. J. Coun. Sci. Ind. Res., v.12, p.50-52, 1939.

HERD, R.P. Control estrategies for ruminant and equine parasites to counter resistance, encystment, and ecotoxicity in the USA. Vet. Parasitol., v.48, p.327336, 1993.

KEITH, R.K. The differentiation on the infective larvae of some common nematode parasites of cattle. Austr. J. Zool., v.1, p.223-235, 1953.

LACKEY, B.A.; MULDOON, A.E.; JAFFE, B.A. Alginate pellet formulation of Hirsutella rossiliensis for biological control of plant-parasitic nematodes. Biol. Cont., v.3, p.155-160, 1993.

LIMA, W.S. Dinâmica das populações de nematódeos parasitos gastrintestinais em bovinos de corte, alguns aspectos da relação parasito-hospedeiro e do comportamento dos estádios de vida livre na região do vale do Rio Doce, MG, Brasil. 1989. 178f. Tese (Doutorado) - Instituto de Ciências Biológicas,
Universidade Federal de Minas Gerais, Belo Horizonte

LIU, X.Z.; ZHANG, K.Q. Nematode-trapping species of Monacrosporium with special reference to two new species. Mycol. Res., v.98, p.862-868, 1994.

MANUELLI, P. R.; WALLER, P. J.; FAEDO, M. et al. Biological control of nematodes parasites of livestock in Fiji: screening of fresh dung of small ruminants for the presence of nematophagous fungi. Vet. Parasitol., v.81, p.39-45, 1999.

PAIVA, F.; SATO M.O.; ACUÑA A.H. et al. Resistência à ivermectina constatada em Haemonchus placei e Cooperia punctata em bovinos. Hora Vet., v.20, p.29-32, 2001.

PANDEY, V.S. Predatory activity of nematode trapping fungi against the larvae of Trichostrongylus axei and Ostertagia ostetargi: A possible method of biological control. J. Helminthol. V.1, p.35-48, 1973.

RABELO, A.M.G. Avaliação $d a$ sobrevivência dos fungos nematófagos Arthrobotrys musiformis $e$ Duddingtonia flagrans após incorporação e armazenamento em suplementos alimentares de ruminantes. 2000. 122f. Tese (Doutorado) - Instituto de Ciências Biológicas, Universidade Federal de Minas Gerais, Belo Horizonte.

SAUMELL, C.A.; PADILHA, T. Influence of weather and time of deposition on sheep faeces colonization by nematophagous fungi in the Mata region of Minas Gerais State, Brazil. Appl. Soil Ecol., v.14, p.63-70, 2000.

VAN OORSCHOT, C.A.N. Taxonomy of the Dactylaria complex. V. A review of Arthrobotrys and allied genera. Stud. Mycol., v.26, p.61-95, 1985.

WALKER, H.L.; CONNICK, W.J. Sodium alginate for production and formulation of mycoherbicides. Weed Sci., v.31, p.333-338, 1983.

WALLER, P.J.; FAEDO, M.; ELLIS, K. The potential of nematophagous fungi to control the free-living stages of nematodes parasites of sheep: towards the development of a fungal controlled release device. Vet. Parasitol.,v.102, p.200-308, 2001.

WALLER, P.J.; LARSEN, M. The role of nematophagous fungi in the biological control of nematode parasites of livestock. Int. J. Parasitol., v.23, p.539-546, 1993.

WILLIAMS, J.C.; MAYHEW, R.L. Survival of infective larvae of the cattle nematodes, Cooperia punctata, Trichostrongylus axei and Oesophagostomum radiatum. Am. J. Vet. Res., v. 28, p. 629-640, 1967. 